\title{
REVISÃO de LITERATURA Prevalência de síndrome das pernas inquietas em pacientes com demência: uma atualização
}

Prevalence of restless legs syndrome in patients with dementia: an update

Rafaela Andreas S. Ribeiro', Lais Fardim Novaes², Maria Clara de Morais Faleiros², Marcos Hortes N. Chagas'

\section{RESUMO}

Objetivo: Realizar uma revisão atualizada para avaliar a prevalência de síndrome das pernas inquietas e/ou movimentos periódicos dos membros em pacientes com demência, além de discutir dados sobre possíveis fatores de risco desses transtornos. Métodos: A revisão foi realizada utilizando a base de dados do PubMed, com o seguinte cruzamento de descritores: (dementia, Alzheimer's disease, dementia with Lewy's body or frontotemporal dementia) e (restless legs syndrome or periodic leg movements). Resultados: Foram selecionados sete artigos, os quais encontraram prevalência de síndrome das pernas inquietas, que variou de $4 \%$ a $24 \%$ em idosos com demência. Houve diferenças importantes entre os métodos utilizados para

\section{Palavras-chave}

Demência, sono, síndrome das pernas inquietas.

\section{Keywords}

Dementia, sleep, restless legs syndrome. o diagnóstico, tamanho das amostras e causas de demência estudadas. Conclusão: Mais estudos com o objetivo de refinar os critérios diagnósticos e definir fatores de risco, especialmente relacionadas a idosos com demência, são necessários. Além disso, deve-se ficar atento a essa comorbidade na prática clínica.

\section{ABSTRACT}

Objective: To perform an updated review to assess the prevalence of restless legs syndrome and/or periodic limb movements in patients with dementia, besides the discussion about possible risk factors of these disorders. Methods: A review was performed using the PubMed database, crossing the following descriptors: (dementia, Alzheimer's disease, dementia with Lewy's body or frontotemporal dementia) and (restless legs syndrome or periodic leg movements). Results: We selected seven articles according to the inclusion and exclusion criteria. The prevalence of restless legs syndrome in elderly patients with dementia ranged from $4 \%$ to $24 \%$. There were important differences in the method used to diagnose, sample size and causes of dementia. Conclusion: More studies are necessary to refine the diagnostic criteria and to define risk factors, especially related to elderly patients with dementia. In addition, the health professionals need to pay attention to this comorbidity in clinical practice.

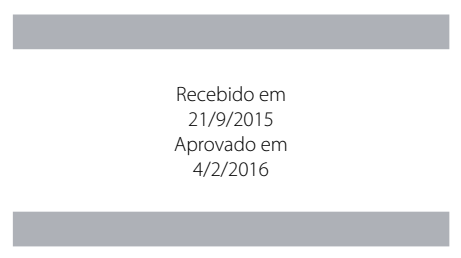

DOI: $10.1590 / 0047-2085000000108$
1 Universidade Federal de São Carlos (UFSCar), Centro de Ciências Biológicas e da Saúde, Departamento de Gerontologia. 2 Universidade de São Paulo (USP), Faculdade de Medicina de Ribeirão Preto, Departamento de Neurociências e Ciências do Comportamento.

Endereço para correspondência: Marcos Hortes N. Chagas

Universidade Federal de São Carlos, Centro de Ciências Biológicas e da Saúde, Departamento de Gerontologia Rodovia Washington Luís, km 235

13565-905 - São Carlos, SP

Telefone: (55 16)3306-6938

E-mails: setroh@hotmail.com; mchagas@ufscar.br 


\section{INTRODUÇÃO}

Os transtornos do sono e queixas relacionadas ao ciclo sono-vigília acometem até 50\% dos indivíduos com mais de 60 anos, resultando em piora na qualidade de vida e no aumento de morbidade e mortalidade'. Em idosos com demência, essas queixas podem ser ainda mais comuns. Recentemente, uma revisão apontou que até 35\% dos pacientes com doença de Alzheimer, causa mais comum de demência, podem apresentar um transtorno de sono ${ }^{2}$. Conjuntamente com o prejuízo cognitivo, alterações de sono como redução do tempo e fragmentação do sono, inquietação e agressividade durante a noite podem gerar dificuldades e sobrecarga aos cuidadores e altos custos para a sociedade ${ }^{2,3}$.

Os mecanismos relacionados aos transtornos de sono em idosos com demência ainda não estão totalmente claros, e vários fatores podem estar envolvidos como o ambiente, genética e outras doenças associadas ${ }^{4}$. Entre os transtornos do sono, a síndrome das pernas inquietas (SPI) e os movimentos periódicos dos membros (MPM) devem ser investigados sistematicamente em virtude de sua alta prevalência na população em geral, especialmente em idosos.

A prevalência da SPI na população aumenta com a idade e é estimada em 5,5\% na população geral e em até 8,7\% na população idosa, sendo mais frequentemente encontrada em pessoas do sexo feminino ${ }^{5}$. No Brasil, Dantas et al. ${ }^{6}$ encontraram a prevalência de 15,6\% em idosos internados em instituições de longa permanência. MPM podem ser encontrados em $5 \%$ a $11 \%$ dos adultos ${ }^{7,8}$ e em até $88 \%$ dos pacientes com a SPI ${ }^{9}$, além de ocorrerem com mais frequência em mulheres, com alta ingesta de cafeína e na presença de estresse e transtornos mentais ${ }^{7,8}$.

A SPI é uma condição que consiste em uma urgência em mover as pernas e que usualmente ocorre com outras sensações anormais como queimação, repuxamento ou como se existissem "insetos rastejando" dentro das pernas. Essas sensações são aliviadas pelo movimento e possuem uma apresentação circadiana piorando à tarde e à noite com alívio significativo pela manhã, independentemente da quantidade de movimento ${ }^{10}$.

Além da idade avançada, a SPI está associada a obesidade, hipertensão arterial sistêmica, roncos, alta ingesta de bebidas alcoólicas, tabagismo e uso de inibidores seletivos da recaptação de serotonina ${ }^{8}$. Nas doenças neurodegenerativas como doença de Parkinson, doença de Alzheimer e demência com corpúsculos de Lewy, é esperado que a prevalência de SPI esteja aumentada, visto que que essas condições estão associadas a perdas neuronais progressivas e alterações em neurotransmissores como a dopamina" ${ }^{11}$.

MPM são caracterizados por clusters de contrações musculares repetitivas nas pernas, causando despertares noturnos. A condição é diagnosticada por meio da realização de polissonografia e da observação de ao menos cinco movimentos por hora de sono, paralelamente, com despertar.

O objetivo desta revisão consistiu em avaliar a prevalência de SPI e/ou MPM em pacientes com demência. Além disso, buscou-se analisar os principais fatores associados a esses transtornos.

\section{MÉTODOS}

A revisão foi realizada utilizando as bases de dados do PubMed, com as seguintes palavras-chave: dementia, Alzheimer's disease, dementia with Lewy's body e frontotemporal dementia, que foram cruzadas uma a uma com restless legs syndrome e periodic leg movements.

Um total de 84 artigos foi encontrado, dos quais foram selecionados sete artigos que tinham como objetivo principal avaliar a associação entre demência e SPI ou MPM. Os critérios de inclusão foram artigos completos, em inglês, sem limite de tempo. Estudos que avaliaram aspectos gerais do ciclo sono-vigília foram excluídos da análise.

\section{RESULTADOS}

A prevalência de SPI em pacientes com demência variou de $4 \%$ a 24\%,3, conforme observado na Tabela 1 . O tamanho das amostras foi de 23 a 431 participantes ${ }^{13,14}$. Em relação à causa de demência, houve grandes diferenças entre os estudos. Apenas um estudo avaliou pacientes apenas com uma causa de demência ${ }^{12}$.

Três estudos selecionados utilizaram a polissonografia para determinar os parâmetros do sono na SPI e MPM $M^{3,13,15}$. A maioria dos estudos que determinou a prevalência de SPI utilizou os critérios do International RLS Study group para o diagnóstico 3,12-14,16.

Em relação ao desenho do estudo, apenas um estudo era longitudinal ${ }^{12} \mathrm{e}$ os demais eram transversais ${ }^{3,4,13-16}$. Três estudos utilizaram idosos saudáveis como grupo controle e também podem ser classificados como caso-controle $e^{4,15,16}$.

A dificuldade dos pacientes em responder às questões elaboradas para identificação da SPI foi investigada por um estudo ${ }^{13}$. O mesmo estudo avaliou pacientes com demência em busca da presença de fatores de risco conhecidos de SPI e encontrou o índice de movimento das pernas ao dormir maior que 15 e o uso de inibidores seletivos da recaptação de serotonina como os mais frequentes ${ }^{13}$.

Em relação aos fatores de risco conhecidos, um estudo ${ }^{14}$ encontrou maior frequência em mulheres, porém outro estudo ${ }^{12}$ apontou para uma taxa maior em homens. Três estudos $3,4,12$ encontraram relação com sintomas neuropsiquiátricos, como agitação noturna, apatia, ansiedade e depressão. 
Tabela 1. Estudos que avaliaram a síndrome das pernas inquietas e o movimento periódico dos membros em pacientes com demência

\begin{tabular}{|c|c|c|c|c|c|}
\hline Estudo & $\mathrm{N}$ & Delineamento & Resultados & Instrumentos & Observaçōes \\
\hline $\begin{array}{l}\text { Talarico et al., } \\
2013^{12}\end{array}$ & 339 pacientes com DA & Coorte prospectiva & $\begin{array}{l}\text { 1. Prevalência de SPI de } 4 \% \\
\text { 2. Frequência maior em } \\
\text { pacientes do sexo masculino } \\
\text { 3. SPI foi associada à presença } \\
\text { de apatia }\end{array}$ & $\begin{array}{l}\text { - MEEM } \\
\text { - Bateria neuropsicológica } \\
\text { - Questionário com critérios } \\
\text { de SPI }\end{array}$ & $\begin{array}{l}\text { SPI parece estar relacionada } \\
\text { com apatia, } 0 \text { que poderia } \\
\text { indicar disfunção do sistema } \\
\text { dopaminérgico central }\end{array}$ \\
\hline $\begin{array}{l}\text { Bhalsing et al., } \\
2013^{16}\end{array}$ & $\begin{array}{l}187 \text { pacientes com } \\
\text { parkinsonismo divididos } \\
\text { em: } \\
134 \text { com DP } \\
5 \text { com demência de Lewy } \\
\text { Outros - } 48 \\
172 \text { sem parkinsonismo }\end{array}$ & Transversal, caso-controle & $\begin{array}{l}\text { 1. Prevalência de SPI de } 9,6 \% \\
\text { no grupo com parkinsonismo } \\
\text { 2. SPI mais frequente em } \\
\text { pacientes com DP - } 11,9 \%\end{array}$ & $\begin{array}{l}\text { - Critérios do International RLS } \\
\text { Study group } \\
\text { - MEEM }\end{array}$ & $\begin{array}{l}\text { Prevalência superior de SPI } \\
\text { em DP em comparação a } \\
\text { controles e outros transtornos } \\
\text { parkinsonianos }\end{array}$ \\
\hline $\begin{array}{l}\text { Bliwise et al., } \\
2012^{15}\end{array}$ & $\begin{array}{l}79 \text { pacientes com } \\
\text { parkinsonismo } \\
28 \text { com DA } \\
187 \text { sem doença } \\
\text { neurodegenerativa } \\
\text { (controles) }\end{array}$ & Transversal, caso-controle & $\begin{array}{l}\text { 1. Pacientes com parkinsonismo } \\
\text { não tratados com levodopa } \\
\text { apresentaram maior índice de } \\
\text { MPM do que os participantes } \\
\text { com DA e controles } \\
\text { 2. Comportamento de chutar } \\
\text { foi associado com maiores } \\
\text { índices de MPM }\end{array}$ & $\begin{array}{l}\text { - PSG } \\
\text { - Questionário de frequência } \\
\text { de inquietação em membros } \\
\text { inferiores }\end{array}$ & $\begin{array}{l}\text { Resultados compatíveis com } \\
\text { hipótese dopaminérgica para } \\
\text { MPM no parkinsonismo }\end{array}$ \\
\hline $\begin{array}{l}\text { Guarnieri et al., } \\
2012^{14}\end{array}$ & $\begin{array}{l}431 \text { pacientes nos } \\
\text { seguintes grupos: } \\
204 \text { com DA } \\
138 \text { com CCL } \\
43 \text { com DV } \\
35 \text { com DFT } \\
21 \text { com DP ou Lewy }\end{array}$ & Transversal & $\begin{array}{l}\text { 1. Prevalência de } 6,1 \% \text { de SPI } \\
\text { 2. Mulheres e pacientes com } \\
\text { DP/Lewy apresentaram mais } \\
\text { SPI } \\
\text { 3. Mais de } 60 \% \text { dos pacientes } \\
\text { com um ou mais distúrbios } \\
\text { do sono }\end{array}$ & $\begin{array}{l}\text { - MEEM } \\
\text { - Bateria de questionários } \\
\text { sobre o sono }\end{array}$ & $\begin{array}{l}\text { Avaliação clínica apurada de } \\
\text { distúrbios do sono deve ser } \\
\text { realizada em pacientes com } \\
\text { comprometimento cognitivo }\end{array}$ \\
\hline Rose et al., $2011^{3}$ & 59 idosos com demência & Transversal & $\begin{array}{l}\text { 1. Prevalência de SPI de } 24 \% \\
\text { 2. Comportamento de agitação } \\
\text { noturna foi relacionado com SPI } \\
\text { 3. Demência mais grave: maior } \\
\text { latência para o sono }\end{array}$ & $\begin{array}{l}\text { - Entrevista com o cuidador } \\
\text { - PSG } \\
\text { - Inventário Cohen-Mansfield } \\
\text { modificado } \\
\text { - MEEM }\end{array}$ & $\begin{array}{l}\text { SPI está associada com } \\
\text { comportamentos de agitação } \\
\text { noturna em pacientes com } \\
\text { demência }\end{array}$ \\
\hline $\begin{array}{l}\text { Rongve et al., } \\
2010^{4}\end{array}$ & $\begin{array}{l}151 \text { idosos com demência } \\
\text { e } \\
420 \text { sem demência }\end{array}$ & $\begin{array}{l}\text { Transversal, } \\
\text { caso-controle }\end{array}$ & $\begin{array}{l}\text { 1. Distúrbios do sono - } 71 \% \text { dos } \\
\text { participantes com demência e } \\
55,7 \% \text { dos controles } \\
\text { 2. Prevalência de SPI de } 20,7 \% \\
\text { 3. Distúrbios do sono mais } \\
\text { frequentes em participantes } \\
\text { com demência de Lewy e DP } \\
\text { do que DA } \\
\text { 4. Distúrbios do sono - } \\
\text { associação com depressão e } \\
\text { ansiedade }\end{array}$ & $\begin{array}{l}\text { - DSM-IV } \\
\text { - Questionário Mayo Sleep } \\
\text { - Inventário Neuropsiquiátrico }\end{array}$ & $\begin{array}{l}\text { Distúrbios de sono são } \\
\text { frequentes em pacientes com } \\
\text { demência, especialmente em } \\
\text { pacientes com demência por } \\
\text { corpos de Lewy }\end{array}$ \\
\hline $\begin{array}{l}\text { Richards et al., } \\
2010^{13}\end{array}$ & 23 idosos com demência & Transversal & $\begin{array}{l}\text { 1. MPM estava presente em } \\
54,55 \% \text { dos pacientes } \\
\text { 2. Uso de ISRS foi o segundo } \\
\text { fator de risco mais presente } \\
(34,78 \%) \\
\text { 3. Comportamentos associados } \\
\text { mais frequentes: maneirismos } \\
(56,52 \%) \text { e inquietação global } \\
(34,78 \%)\end{array}$ & $\begin{array}{l}\text { - PSG } \\
\text { - Observação direta } \\
\text { - Entrevista clínica com } \\
\text { critérios diagnóstico de SPI }\end{array}$ & $\begin{array}{l}\text { Idosos com demência inicial } \\
\text { a moderada mostraram-se } \\
\text { incapazes de entender e } \\
\text { responder ao questionário para } \\
\text { diagnóstico de SPI }\end{array}$ \\
\hline
\end{tabular}

MPM: movimento periódico dos membros; SPl: síndrome das pernas inquietas; DP: doença de Parkinson; DA: doença de Alzheimer; CLL: comprometimento cognitivo leve; DV: demência vascular; SAOS: síndrome da apneia obstrutiva do sono; PSG: polissonografia; ISRS: inibidores seletivos da recaptaçăo de serotonina; MEEM: Miniexame do Estado Mental; DSM: Manual Diagnóstico e Estatístico dos Transtornos Mentais.

\section{DISCUSSÃO}

Nesta revisão, a prevalência de SPI variou entre $4 \%^{12}$ e $24 \%{ }^{3}$. Essa ampla variação pode ser explicada pela heterogeneidade das populações estudadas nos diferentes estudos (pacientes com quadros demenciais iniciais a graves, diferentes etiologias de demência), variação no método diagnóstico utilizado (questionários específicos, observação direta, relato dos pacientes ou dos cuidadores), presença ou não de fatores de risco para SPI, entre outros. Essa constatação pode representar uma limitação para a interpretação dos dados obtidos. 
Em relação aos possíveis fatores associados à SPI, nenhum estudo avaliou de maneira sistemática o impacto de possíveis causas na taxa de prevalência de SPI e MPM em pacientes com demência. O fato de os estudos terem desenhos transversais ou serem caso-controles, exceto o estudo de Talarico et al. ${ }^{12}$, impede a avaliação de causa e efeito em relação a possíveis fatores de risco. Os sintomas e sinais da SPI e/ou MPM poderiam ser secundários ou estar associados aos seguintes fatores: uso frequente de cafeína, distúrbios psiquiátricos, deficiência de ferro, doença renal terminal, doenças reumatológicas, diabetes mellitus, doença pulmonar obstrutiva crônica, síndrome da apneia obstrutiva do sono, câncer, uso de bloqueadores dopaminérgicos, uso de inibidores da recaptação de serotonina e doenças neurológicas ${ }^{8,11}$.

Três estudos dividiram os participantes em grupos de acordo com a causa da demência e a doença neurodegenerativa ${ }^{14-16}$. É interessante notar que dois estudos encontram prevalência aumentada em pacientes com parkinsonismo comparados com outros grupos, reforçando a hipótese de a etiologia da SPI estar relacionada à disfunção do sistema dopaminérgico ${ }^{15,16}$. Além disso, Talarico et al. ${ }^{12}$ encontraram a relação entre SPI e apatia em pacientes com DA. Sabe-se que sintomas de apatia estão relacionados com áreas cerebrais ligadas ao mecanismo de recompensa, que são mediadas pelo sistema dopaminérgico5. Por fim, o estudo de Bliwise et al. ${ }^{15}$ evidenciou ainda que pacientes com parkinsonismo tratados com levodopa, idosos sem parkinsonismo e idosos com DA apresentaram menores índices de MPM do que pacientes com parkinsonismo que não estavam recebendo levodopa, mais uma vez reforçando a hipótese do envolvimento de vias dopaminérgicas na fisiopatologia da SPI e MPM.

Ainda em relação à fisiopatologia, a deficiência de ferro é frequentemente associada à SPI e aos MPM, possivelmente devido ao seu envolvimento com o sistema dopaminérgico, visto que o ferro age como cofator na via de produção de dopamina. Apesar disso, Berger et al. ${ }^{17}$ não encontraram relação entre os níveis de ferritina e transferrina e a prevalência de SPI na população idosa. Além disso, essa associação não foi adequadamente explorada nos estudos selecionados.

Richards et al..$^{13}$ destacaram a dificuldade dos pacientes com demência em responder às questões elaboradas, evidenciando a limitação da entrevista diagnóstica atualmente disponível, comprometendo sua confiabilidade e sensibilidade. Mesmo pacientes com demência leve apresentam habilidades cognitivas e de comunicação prejudicadas para o fornecimento de informações necessárias para o diagnóstico, visto que a entrevista diagnóstica subjetiva exige um recordatório complexo dos sintomas da SPI. Dessa forma, há necessidade de elaboração de um método diagnóstico de maior sensibilidade para a detecção de ambas as condições ${ }^{13}$, visto que a entrevista feita diretamente com o paciente pa- rece ser insuficiente para o diagnóstico. Uma alternativa é a administração de questionários e entrevistas também aos cuidadores, o que poderia aumentar a sensibilidade na detecção de transtornos de sono ${ }^{18}$.

A dificuldade na realização desses diagnósticos pode ser atribuída a um conjunto heterogêneo de fatores, entre eles: (i) não investigação de tais condições e de seus fatores de risco por parte dos profissionais de saúde; (ii) dificuldade dos pacientes em relatar os sintomas que possibilitem o diagnóstico clínico; (iii) sensibilidade baixa do método diagnóstico utilizado; (iv) inacessibilidade a procedimentos que visem ao esclarecimento diagnóstico (polissonografia); e (v) confusão com diagnósticos diferenciais (acatisia, neuropatias) ${ }^{11,13}$.

A observação de critérios adicionais ou de suporte para auxílio na elaboração do diagnóstico de SPI poderia ser útil em idosos com ou sem demência. Os seguintes sinais e sintomas devem ser destacados: resposta a agentes dopaminérgicos, história familiar de SPI, histórico detalhado obtido de cuidadores e/ou familiares, qualidade de sono superior durante o dia, história pregressa sugestiva da síndrome, MPM durante vigília ou observados em polissonografia, distúrbios do sono comórbidos, necessidade de contenção física à noite, níveis reduzidos de ferritina, doença renal terminal, diabetes, neuropatia ou radiculopatia ${ }^{11,13,19}$.

Vale salientar que a dificuldade do diagnóstico de SPI é um problema enfrentado não somente na presença de declínio cognitivo. Hening et al. ${ }^{20}$ mostraram que apenas 12,9\% dos pacientes que consultaram um médico na atenção básica por causa de sintomas de SPI receberam o diagnóstico. Na mais recente atualização dos critérios diagnósticos pelo International Restless Legs Syndrome study group, a dificuldade de padronização de critérios válidos para diagnóstico no idoso é destacada ${ }^{21}$. Dessa forma, deve-se ter especial atenção aos sintomas principais e de suporte em idosos com demência, visando ao diagnóstico correto e ao tratamento adequado.

\section{CONCLUSÃO}

Novos estudos são necessários para validação e refinamento dos critérios diagnóstico da SPI e MPM na demência, assim como para explorar as particularidades dessa associação. Com uma população geriátrica crescente, o reconhecimento adequado dessas condições tão prevalentes faz-se necessário. Assim, estratégias de rastreamento poderiam ser adotadas para identificação desses transtornos, buscando melhorar a qualidade de vida dos pacientes e de seus cuidadores. Entre as estratégias adicionais, a entrevista com familiar e a busca também pelos sintomas de suporte devem ser destacadas. 


\section{CONTRIBUIÇÕES INDIVIDUAIS}

Rafaela Andreas S. Ribeiro - Contribuiu na escrita, revisão dos artigos e finalização do manuscrito.

Lais Fardim Novaes e Maria Clara de Morais Faleiros Contribuíram na primeira versão do manuscrito, revisão dos artigos e busca dos artigos.

Marcos Hortes N. Chagas - Supervisionou a busca de artigos e contribuiu na escrita e finalização do manuscrito.

\section{CONFLITOS DE INTERESSE}

Todos os autores declaram não apresentar conflitos de interesse.

\section{REFERÊNCIAS}

1. Neikrug $A B$, Ancoli-Israel S. Sleep disorders in the older adult - a mini-review. Gerontology. 2010;56(2):181-9.

2. Dauvilliers Y. Insomnia in patients with neurodegenerative conditions. Sleep Med. 2007;8 Suppl 4:S27-34.

3. Rose KM, Beck C, Tsai PF, Liem PH, Davila DG, Kleban M, et al. Sleep disturbances and nocturnal agitation behaviors in older adults with dementia. Sleep. 2011;34(6):779-86.

4. Rongve A, Boeve BF, Aarsland D. Frequency and correlates of caregiver-reported sleep disturbances in a sample of persons with early dementia. J Am Geriatr Soc. 2010;58(3):480-6.

5. Schultz W, Dayan P, Montague PR. A neural substrate of prediction and reward. Science. 1997;275(5306):1593-9.

6. Dantas FG, Medeiros JLA, Farias KS, Ribeiro CD. Restless legs syndrome in institutionalized elderly. Arq Neuropsiquiatr. 2008;66(2):328-30.

7. Hornyak M, Feige B, Riemann D, Voderholzer U. Periodic leg movements in sleep and periodic limb movement disorder: prevalence, clinical significance and treatment. Sleep Med Rev. 2006;10(3):169-77.

8. Ohayon MM, Roth T. Prevalence of restless legs syndrome and periodic limb movement disorder in the general population. J Psychosom Res. 2002:53(1):547-54.
9. Montplaisir J, Boucher S, Poirier G, Lavigne G, Lapierre O, Lespérance P. Clinical, polysomnographic, and genetic characteristics of restless legs syndrome: a study of 133 patients diagnosed with new standard criteria. Mov Disord. 1997;12(1):61-5.

10. Salas RE, Gamaldo CE, Allen RP. Update in restless legs syndrome. Curr Opin Neurol. 2010;23(4):401-6.

11. Hornyak M, Trenkwalder C. Restless legs syndrome and periodic limb movement disorder in the elderly. J Psychosom Res. 2004:56(5):543-8.

12. Talarico G, Canevelli M, Tosto G, Vanacore N, Letteri F, Prastaro M, et al. Restless legs syndrome in a group of patients with Alzheimer's disease. Am J Alzheimers Dis Other Demen. 2013;28:165-70.

13. Richards K, Shue VM, Beck CK, Lambert CW, Bliwise DL. Restless legs syndrome risk factors, behaviors, and diagnoses in persons with early to moderate dementia and sleep disturbance. Behav Sleep Med. 2010;8(1):48-61.

14. Guarnieri B, Adorni F, Musicco M, Appollonio I, Bonanni E, Caffarra P, et al. Prevalence of sleep disturbances in mild cognitive impairment and dementing disorders: a multicenter Italian clinical cross-sectional study on 431 patients. Dement Geriatr Cogn Disord. 2012;33(1):50-8

15. Bliwise DL, Trotti LM, Yesavage JA, Rye DB. Periodic leg movements in sleep in elderly patients with Parkinsonism and Alzheimer's disease. Eur J Neurol. 2012:19(6):918-23.

16. Bhalsing K, Suresh K, Muthane UB, Pal PK. Prevalence and profile of Restless Legs Syndrome in Parkinson's disease and other neurodegenerative disorders: a case-control study. Parkinsonism Relat Disord. 2013;19(4):426-30.

17. Berger K, von Eckardstein A, Trenkwalder C, Rothdach A, Junker R, Weiland SK. Iron metabolism and the risk of restless legs syndrome in an elderly general population - the MEMO-Study. J Neurol. 2002;249(9):1195-9.

18. McCurry SM, Vitiello MV, Gibbons LE, Logsdon RG, Teri L. Factors associated with caregiver reports of sleep disturbances in persons with dementia. Am J Geriatr Psychiatry. 2006;14(2):112-20.

19. Allen RP, Picchietti D, Hening WA, Trenkwalder C, Walters AS, Montplaisi J; Restless Legs Syndrome Diagnosis and Epidemiology workshop at the National Institutes of Health; International Restless Legs Syndrome Study Group. Restless legs syndrome: diagnostic criteria, special considerations, and epidemiology. A report from the restless legs syndrome diagnosis and epidemiology workshop at the National Institutes of Health. Sleep Med. 2003:4(2):101-19.

20. Hening W, Walters AS, Allen RP, Montplaisir J, Myers A, Ferini-Strambi L. Impact, diagnosis and treatment of restless legs syndrome (RLS) in a primary care population: the REST (RLS epidemiology, symptoms, and treatment) primary care study. Sleep Med. 2004;5(3):237-46.

21. Allen RP, Picchietti DL, Garcia-Borreguero D, Ondo WG, Walters AS, Winkelman JW, et al.; International Restless Legs Syndrome Study Group. Restless legs syndrome/Willis-Ekbom disease diagnostic criteria: updated International Restless Legs Syndrome Study Group (IRLSSG) consensus criteria - history, rationale, description, and significance. Sleep Med. 2014;15(8):860-73 